\title{
HIERARCHICAL JOINT REGISTRATION OF TISSUE BLOCKS WITH SOFT SHAPE CONSTRAINTS FOR LARGE-SCALE HISTOLOGY OF THE HUMAN BRAIN
}

\author{
M. Mancini ${ }^{1}$, S. Crampsie ${ }^{2}$, D.L. Thomas ${ }^{3,4}$, Z. Jaunmuktane ${ }^{2}$, J.L. Holton ${ }^{2}$, J.E. Iglesias ${ }^{1}$ \\ ${ }^{1}$ Center for Medical Image Computing, Dept. Med. Phys. \& Biomed. Eng., University College London \\ ${ }^{2}$ Queen Square Brain Bank, Institute of Neurology, University College London \\ ${ }^{3}$ Neuroradiological Academic Unit, Institute of Neurology, University College London \\ ${ }^{4}$ Leonard Wolfson Experimental Neurology Centre, Institute of Neurology, University College London
}

\begin{abstract}
Large-scale 3D histology reconstruction of the human brain with MRI as volumetric reference generally requires reassembling the tissue blocks into the MRI space, prior to any further reconstruction of the histology of the individual blocks. This is a challenging registration problem, particularly in the frequent case that blockface photographs of paraffin embedded tissue are used as intermediate modality, as their contrast between white and gray matter is rather low. Here we propose a registration framework to address this problem, relying on two key components. First, blocks are simultaneously aligned to the MRI while exploiting the spatial constraints that they impose on each other, by means of a customized soft shape constraint (similarly to a jigsaw puzzle). And second, we adopt a hierarchical optimization strategy that capitalizes on our prior knowledge on the slicing and blocking procedure. Our framework is validated quantitatively on synthetic data, and qualitatively on the histology of a whole human hemisphere.
\end{abstract}

Index Terms - Registration, ex vivo MR, histology, brain

\section{INTRODUCTION}

Histology and magnetic resonance imaging (MRI) are two powerful tools to study biological systems, in particular the brain. However, they target very different scales: on the one hand, histology allows microstructural examination of small samples with high levels of detail; on the other hand, MRI offers a global overview of the entire brain. These complementary approaches hold great potential in building wholebrain atlases with histology-level resolution, the goal of several large-scale histology initiatives [1,2].

Building such atlases presents several challenges. First, the fact that histological analysis yields a set of spatially distorted 2D sections, due to cutting, processing, sectioning, and mounting of tissue. Recovering the 3D structure of histological sections, which often involves registration to a reference volume (typically MRI), is a difficult, largely studied problem in the literature [3]. A related challenge is the large differences in contrast and resolution between MRI and histology, which, along with histological artifacts (e.g., tears, folding) make registration between the two modalities difficult. Another obstacle in large-scale histology studies of the human brain is the size of the human brain, which requires tailored whole-brain microtomes that are only available in very few selected sites around the world.

An alternative to whole-brain microtomes is cutting the brain into blocks, which enables tissue processing with standard equipment and techniques - and hence applicability at nearly every research site. The cutting procedure is constrained by both the necessity to preserve structures of interest (e.g., hippocampus [4], subthalamic nuclei [5]), and the practical limits imposed by the standard size of cassettes and glass slides (i.e., a maximum of $74 \times 52 \mathrm{~mm}$ ), for subsequent tissue processing, sectioning and staining. A resulting issue is hence the need to solve a "jigsaw puzzle" to reassemble the blocks, before further reconstruction of individual blocks.

The registration between blocks and MRI is commonly carried out by means of an intermediate modality, which does not suffer from nonlinear geometric distortions, most frequently blockface photographs. The literature on MRIblockface registration is rather sparse. A frequent approach is to align each volume of blockface photographs to the MRI independently. However, this registration is not easy (despite its linear nature, and even with reasonable initialization) because of its whole-to-part nature, particularly when the contrast of the blockface images is poor - which is the case for the ubiquitous paraffin embedding.

In this paper, we present a hierarchical approach to the blockface-MRI registration problem, which jointly registers all blocks, while ensuring that they do not overlap or leave gaps in between (i.e., the pieces in the jigsaw puzzle fit). This is achieved by a soft constraint imposed on the shapes of the blocks, and which is integrated in the loss function of the registration. The optimization is carried out in a hierarchical fashion, which takes advantage of our prior knowledge on the geometry of the brain cut and blocking procedures. 


\section{METHODS}

\subsection{General registration framework}

Let $V(\boldsymbol{x})$ be a volumetric MRI scan, defined in a spatial domain $\boldsymbol{x} \in \Omega_{V}$. Likewise, let $\left\{B_{n}(\boldsymbol{x})\right\}_{n=1, \ldots, N}$ be $N$ volumes of blockface photographs to be registered to the MRI scan, defined in their own domains $\left\{\Omega_{n}\right\}$. In addition, we assume the availability of binary masks $M_{V}(\boldsymbol{x})$ and $\left\{M_{n}(\boldsymbol{x})\right\}_{n=1, \ldots, N}$ for the MRI scan and blockface volumes, respectively, where $M_{V}, M_{n} \in\{0,1\}$. These masks are assumed to be obtained with an automated segmentation algorithm, and hence to be noisy. Our goal is then to find a set of linear spatial transforms, parameterized by their $4 \times 4$ transformation matrices $\left\{\Theta_{n}\right\}_{n=1, \ldots, N}$, that best align the blocks. This is achieved by minimizing the following general loss function:

$$
\begin{aligned}
& \mathcal{L}=\frac{1}{N} \sum_{n=1}^{N} \mathcal{D}\left[V(\boldsymbol{x}), B_{n}\left(T\left(\boldsymbol{x}, \Theta_{n}\right)\right)\right]+\frac{\gamma_{1}}{N} \sum_{n=1}^{N} \mathcal{R}\left(\Theta_{n}\right) \\
& +\gamma_{2} \mathcal{C}\left[M_{V}(\boldsymbol{x}), M_{1}\left(T\left(\boldsymbol{x}, \Theta_{1}\right)\right), \ldots, M_{N}\left(T\left(\boldsymbol{x}, \Theta_{N}\right)\right)\right]
\end{aligned}
$$

where $\mathcal{D}$ is a dissimilarity metric between the MRI and each of the registered blocks; $\mathcal{R}$ is a regularizer that encodes prior knowledge on the transforms; $\mathcal{C}$ is a soft constraint term that penalizes implausible spatial arrangement of the blockface volumes based on their masks; and $\gamma_{1}, \gamma_{2}$ are relative weights.

\subsection{Modeling of spatial constraints with $\mathcal{C}$}

The term $\mathcal{C}$ encodes our prior knowledge on the spatial relationships between the different blocks. If the blockface volumes were perfectly cut (i.e, without any deformation) and segmented, then we would expect them to fit exactly, and could use hard constraints to prevent them from overlapping or being disjoint. In practice, this is never the case, so we resort to a soft, continuous regularizer, which simultaneously penalizes overlap and gaps, while encouraging the external boundary of the blocks to fit that of the MRI:

$$
\mathcal{C}=\sum_{\boldsymbol{x} \in \Omega_{V}}\left|M_{V}(\boldsymbol{x})-\sum_{n} M_{n}\left[T\left(\boldsymbol{x}, \Theta_{n}\right)\right]\right| .
$$

When a gap between blocks exists at a given voxel, the sum of masks is zero, and a penalty of one is incurred. When two masks overlap at a voxel, the sum is two, and a penalty of one is incurred as well. Higher penalty is paid when multiple blocks overlap at the same voxel. Also, by considering all voxels inside the MRI mask in the sum, penalty is also incurred when the union of the blockface masks does not match $M_{V}(\boldsymbol{x})$ well, effectively fitting the outer boundaries of the blocks and the MRI mask. We note that we also tried a quadratic function in Eq. 2, but more robust results were obtained with the $L_{1}$ norm in pilot experiments.

\subsection{Hierarchy}

Rather than optimizing all $\left\{\Theta_{n}\right\}$ directly in Equation 1, an alternative is force groups of transforms to share the same parameters in the earlier iterations of the algorithm, to reflect our prior knowledge on the cutting procedure. For instance, one could group the blocks from the same slice in the first levels, and then allow each block to have its own transform in finer levels.

This hierarchy can be reflected by replacing $\Theta_{n}$ by $\Theta_{j(n ; h)}^{h}$ in Eqns. 1 and 2, where $j(n ; h)$ groups the blocks that share parameters $\Theta_{j}^{h}$ at hierarchy level $h$ (where $h=$ $1, \ldots, H)$. We use $J_{h}$ to refer to the number of different transforms at hierarchy level $h$, such that $J_{h}<J_{h+1}$, and (typically) $J_{H}=N$. We also note that the number of parameters of $\Theta_{j}^{h}$ can be different at every level, e.g., could increase from 3 (translation transform) to 6 (rigid), 7 (similarity) or 12 (affine), as we progress through the hierarchy.

\subsection{Model instantiation and implementation details}

We complete the framework with choices for the dissimilarity metric $\mathcal{D}$ and the regularizer $\mathcal{R}$. For the dissimilarity, we use the negated cross correlation between the edge maps (gradient magnitudes) of the two images. Even though mutual information might be a more natural alternative, our choice proved to be more robust and perform better in preliminary experiments. For the regularizer, we first computed the $\log$ Jacobian linear transformation matrices, and quadratically penalized deviations from an a priori value $\eta$ that describes the expected shrinkage due to tissue processing (which can be determined empirically):

$$
\mathcal{R}\left(\Theta_{n}\right)=\left[\log \left|\Theta_{n}\right|-\eta\right]^{2} .
$$

We can then combine Eqns. 1-3 to obtain the final loss function at hierarchy level $h$ :

$$
\begin{aligned}
\mathcal{L}= & -\frac{1}{N} \sum_{n=1}^{N} N C C\left[V^{\mathcal{E}}(\boldsymbol{x}), B_{n}^{\mathcal{E}}\left(T\left(\boldsymbol{x}, \Theta_{j(n ; h)}^{h}\right)\right)\right] \\
& +\frac{\gamma_{1}}{J_{h}} \sum_{j=1}^{J_{h}}\left(\log \left|\Theta_{j(n ; h)}^{h}\right|-\eta\right)^{2} \\
& +\gamma_{2} \sum_{\boldsymbol{x} \in \Omega_{V}}\left|M_{V}(\boldsymbol{x})-\sum_{n} M_{n}\left[T\left(\boldsymbol{x}, \Theta_{j(n ; h)}^{h}\right)\right]\right|,
\end{aligned}
$$

where $V^{\mathcal{E}}$ and $\left\{B_{n}^{\mathcal{E}}\right\}$ denote the edge maps of the MRI volume and blocks, respectively, and NCC is the normalized cross correlation. In order to minimize Eq. 4, we use the LBFGS algorithm [6]. We note that we use linear interpolation for $M_{n}\left[T\left(\boldsymbol{x}, \Theta_{j(n ; h)}^{h}\right)\right]$, otherwise derivatives would be zero almost everywhere. 


\section{EXPERIMENTS AND RESULTS}

\subsection{Data}

\subsubsection{Real data}

We used a left human hemisphere donated for research to the UCL Queen Square Brain Bank, through a program with approval from Research Ethics Committes. MRI data were acquired ex vivo with a T2 CISS sequence (TR $=6.37 \mathrm{~ms}$, $\mathrm{TE}=2.88 \mathrm{~ms}, \mathrm{BW}=449 \mathrm{~Hz} / \mathrm{Px}$, ten averages), with Fluorinert immersion to avoid susceptibility artifacts. For the histological processing, the hemisphere was first separated into cerebrum, cerebellum and brainstem. Each of them was subsequently cut into $10 \mathrm{~mm}$ thick slices, following the coronal, sagittal and axial directions, respectively. The cerebrum slices were further cut in blocks able to fit large cassettes $(74 \times 52 \mathrm{~mm})$, while preserving the main subcortical structures and taking pictures before and after this cut (brain cut photographs). Each block was then processed and embedded in paraffin. The blocks were sectioned at $25 \mu \mathrm{m}$ thickness, while taking blockface photographs of every section. The blockface photographs were stacked in volumes, corrected for perspective [3], and co-registered with the SIFT algorithm [7]. The rearranging of the blockface volumes was initialized with registrations derived from the brain cut photographs.

\subsubsection{Synthetic data}

Synthetic data were used to evaluate the registration method quantitatively, as obtaining perfectly aligned MRI and blockface volumes is extremely challenging. Here we used 50 randomly selected multimodal (T1/T2) scans from the IXI dataset (brain-development.org/ixi-dataset/), letting the $\mathrm{T} 1$ play the role of reference $(V)$, and deforming the T2 to play the role of blockface volumes $\left(\left\{B_{n}\right\}\right)$.

The synthetic deformation mimicked the brain cut procedure as follows: 1. Segmentation of the T1 with FreeSurfer [8] to extract the left hemisphere and subdivide it into cerebrum, cerebellum and brainstem; the union of these three masks, corrupted with noise at the boundary, provides the mask $M_{V}$, and the background of the T1 volume is corrupted by Gaussian noise. 2. Subdivision of the cerebrum, cerebellum and brainstem of the T2 into $10 \mathrm{~mm}$ thick slices, with coronal, sagittal, and axial orientation, respectively. 3. Further subdivision of cerebrum slices into blocks, using a sampling procedure to attempt to minimize both the number of blocks (constrained to fit in a $74 \times 52 \mathrm{~mm}$ cassette) and the number of crossed structure boundaries. 4. Corrupting blocks and corresponding masks the same way as the T1. 5. Corrupting the positioning of the blocks with random similarity transforms, first in groups corresponding to slices, and then each block individually - attempting to reproduce the type of initialization that one typically achieves with brain cut photographs. Sample T1 and corrupted T2 volumes can be seen in Fig. 1a,b.

\subsection{Experimental setup}

For the quantitive evaluation, we attempted to recover the original location of the T2 blocks by minimizing Eq. 4 with four competing approaches. The baseline approach consisted of independent registration of the blocks, i.e., with $\gamma_{2}=0$ and no hierarchy. The second approach was the full model with $\gamma_{2}>0$, and a hierarchy with four levels: 1 . Blocks grouped in slices, allowed to translate and rotate only on the plane parallel to the slice; 2 . Blocks may individually translate and rotate on the plane parallel to the block, and a global scaling factor is shared among blocks; 3 . Same as 2, but each block has its own scaling factor; and 4 . Full similarity transform for each block. Finally, we attempted to separate the contributions of the hierarchy and the soft spatial constraints by evaluating a model with $\gamma_{2}>0$ by only the finest hierarchy level ("Overlap"), and a model with full hierarchy but $\gamma_{2}=0$ ("Hierarchy"). Models were evaluated with statistics on the registration error (in $\mathrm{mm}$ ) across all voxels in the masks of the $\mathrm{T} 2$ blocks.

Throughout the experiments, we used the following parameters: $\gamma_{1}=1, \gamma_{2}=1$ (or 0 if for the baseline and "Hierarchy"), and $\eta=\log (3 / 4)$ - which was empirically determined to be the approximate shrinking factor due to tissue processing in our setup. The full model with $\gamma_{1}=\gamma_{2}=1$ was used for the real data. In all cases, we operated at $1 \mathrm{~mm}$ resolution, but the resulting linear transforms can be applied to the original data at any resolution.

\subsection{Results on synthetic data}

Table 1 shows the mean, standard deviation, and robust maximum $\left(95^{\text {th }}\right.$ percentile) of the registration error across the 50 volumes, for the four competing methods. Using the hierarchy alone provides modest reductions $(\sim 5 \%)$ on the mean and maximum errors. When spatial constraints are used with no hierarchy, larger improvement is achieved with respect to the baseline, both in terms of mean and maximum errors. When the hierarchy is added on top of spatial constraints, the mean error barely decreases, but the robustness of the method fairly improves, as reflected by the reductions in the standard deviation of the error and, especially, its robust maximum (0.4 $\mathrm{mm}$ decrease). Compared with the baseline method, the full model achieves a $14 \%$ reduction in mean error and $26 \%$ decrease in maximum error, thanks to the robustness provided by the spatial constraints. Sample reconstructions produced by the different methods are shown in Figs. 1 and 2.

\subsection{Results on real data}

Figure 3 shows the results obtained on the real data. Even with the already mentioned differences between MRI and blockface photographs, the final alignment between the two modalities shows good qualitative details, e.g. the alignment of the ventricle, the smooth separation between cerebellum and cerebrum. 


\begin{tabular}{|c|c|c|c|}
\hline Method & Mean & Std. dev. & Robust Max. \\
\hline Baseline & 2.15 & 1.11 & 4.48 \\
Hierarchy & 2.08 & 1.08 & 4.25 \\
Overlap & 1.86 & 0.93 & 3.72 \\
Full model & 1.85 & 0.82 & 3.34 \\
\hline
\end{tabular}

Table 1. Distance error statistics for each method, in $\mathrm{mm}$. The robust maximum is the $95^{t h}$ percentile of the error.

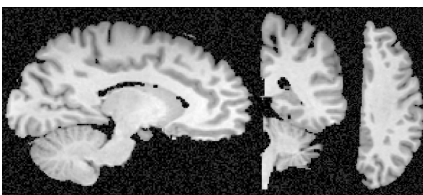

(a)

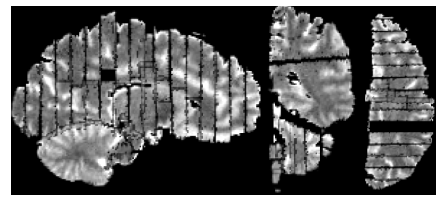

(c)

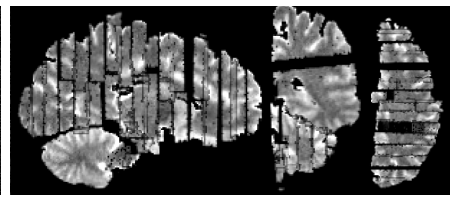

(b)

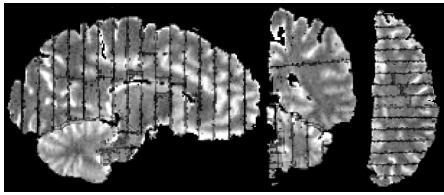

(d)
Fig. 1. Sample sagittal, coronal and axial slices of: (a) Reference T1 volume; (b) Distorted T2 blocks; (c) T2 blocks reconstructed with baseline method; and (3) Reconstructed with proposed (full) model. Notice how our model is able, e.g., to recover the largely displaced block above the occipital horn of the lateral ventricle.

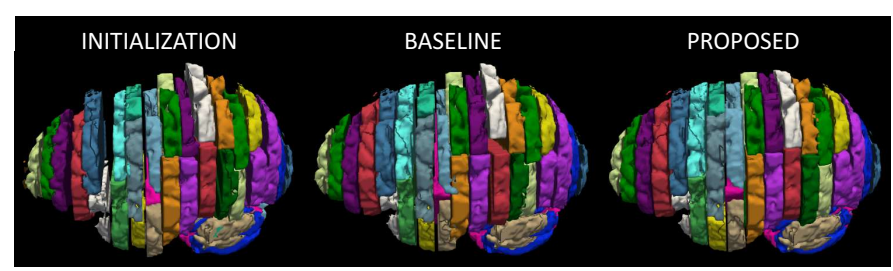

Fig. 2. 3D renderings of the block masks $\left\{M_{n}\right\}$ corresponding to the synthetic blockface volumes from Fig. 1. Note the improvement in, among others, the block mentioned in the caption of the aforementioned figure, displayed in white here.

\section{CONCLUSION}

A method for jointly registering histological volumes to an $e x$ vivo MRI scan by means of blockface photographs has been proposed in this paper. Future work will be focused on a multiresolution version of the algorithm, and on evaluation on real data using pairs of corresponding, manually placed landmarks, or using manual segmentations.

This method will be used as part of a pipeline for full histological reconstruction for whole brain hemispheres, eventually leading to the construction of a high-resolution computational atlas of the human brain based on 3D reconstructed histology.

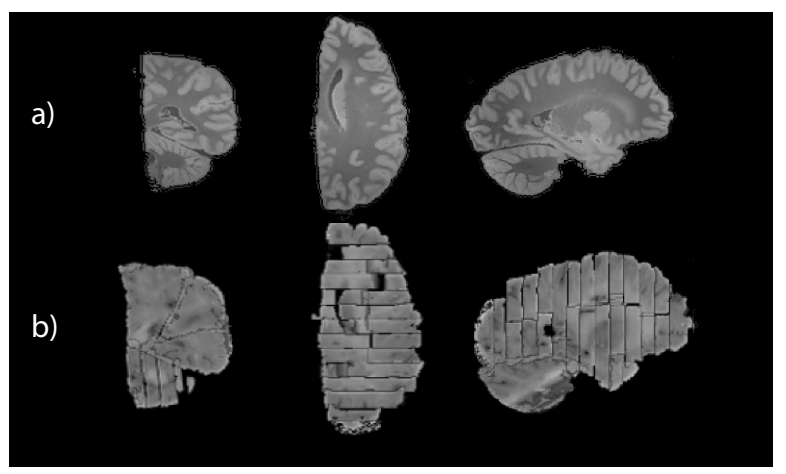

Fig. 3. Sample sagittal, coronal and axial slices of: (a) Reference T2 volume; (b) Reconstructed blockface volume. Notice how most structures, in particular subcortical ones, present a smooth profile across blocks.

Acknowledgement: Research primarily supported by the European Research Council (ERC) through the Starting Grant No 677697 (project "BUNGEE-TOOLS"), awarded to JEI.

\section{REFERENCES}

[1] K. Amunts, C. Lepage, L. Borgeat, et al., "BigBrain: An ultrahigh-resolution 3D human brain model," Science, vol. 340, no. 6139, pp. 1472-1475, 2013.

[2] S. Ding, J.J. Royall, S.M. Sunkin, et al., "Comprehensive cellular-resolution atlas of the adult human brain," $J$. Comp. Neurol., vol. 524, no. 16, pp. 3127-3481, 2016.

[3] J. Pichat, J.E. Iglesias, T. Yousry, et al., "A survey of methods for 3D histology reconstruction," Medical Image Analysis, vol. 46, pp. 73-105, 2018.

[4] J.E. Iglesias, J.C. Augustinack, K. Nguyen, et al., "A computational atlas of the hippocampal formation using ex vivo, ultra-high resolution MRI: Application to adaptive segmentation of in vivo MRI," NeuroImage, vol. 115, pp. 117 - 137, 2015.

[5] L.A. Massey, M.A. Miranda, L. Zrinzo, et al., "High resolution MR anatomy of the subthalamic nucleus: Imaging at 9.4T with histological validation," NeuroImage, vol. 59, no. 3, pp. 2035 - 2044, 2012.

[6] D.C. Liu and J. Nocedal, "On the limited memory BFGS method for large scale optimization," Mathematical programming, vol. 45, no. 1-3, pp. 503-528, 1989.

[7] D.G. Lowe, "Object recognition from local scale invariant features," in Proc. ICCV, 1999, vol. 2, pp. 1150-1157.

[8] B. Fischl, D. Salat, E. Busa, et al., "Whole brain segmentation: automated labeling of neuroanatomical structures in the human brain," Neuron, vol. 33, pp. 341-355, 2002. 